\title{
Mathematical Model for Thin-walled Corrugated Tube under Axial Compression
}

\author{
Arameh Eyvazian ${ }^{1}$ \\ ${ }^{1}$ Mechanical and Industrial Engineering Department, College of Engineering, Qatar University, P.O. Box 2713, Doha, Qatar
}

\begin{abstract}
In this research, theoretical investigation of corrugated aluminum tubes is performed to predicting the energy absorption characteristics. Aim to deform plastic tubes in predetermined intervals, corrugations are introduced on its surface. Theoretical relations are presented for predicting the energy absorption and mean crushing load of corrugated tubes. Other than that, corrugation helps to control the failure mode.
\end{abstract}

\section{Introduction}

Safety is one of the most important issues in the modern design of vehicles, aircrafts and ships. Many researchers try to study structures crashworthiness. These studies are about different geometry of energy absorbing components, their materials and different loading conditions [1]. The loading condition can be quasi-static or dynamic loading. Its applications range from reliability and safety of vehicle transportation to missile delivery, satellite recovery, aircraft soft-landing, etc. [2-4]. Among all the energy absorbing members, thin-walled metal tubes have been more popular due to their low cost, ease of fabrication and excellent energy absorption efficiency. Kinetic energy has been absorbed by thin wall tubes by performing plastic deformation along tube wall. The main aim to use energy absorbers in moving vehicles are protection of people, structures or equipment. Many experimental, numerical, and theoretical studies related to this topic have appeared in the literature (e.g. [1-5]). In order to improve the energy absorption characteristics of such thin-walled tubes, many tests have been conducted on cross-section shapes of tubes $[5,8]$.

Among different type of improves thin walled structures for energy absorption purpose, corrugated tubes catch more attention by researchers in recent years. The metallic or composite tubes with corrugated surface in the axial and radial directions were studied and reported in literature [6-11]. The effects of corrugation on the energy-absorbing characteristics for circular tubes under lateral loading were investigated based on the results of experimental tests [11].

Few research works have been carried out to study the methods of controlling the deformation mechanisms and this field of study still needs more development.

In this paper, Theoretical relation for predicting mean crushing load and absorbed energy has been developed.

\section{Theoretical formulations}

Alexander's theoretical model for prediction of mean load for tubular energy absorbers under axial load is still one of popular models with designers and scientists.

Other more advance models are available now. Compare to these models in the same time, complicated and configurations, Alexander's model can predict the mean load with better approximation. Therefore, after many decades that passed from introducing, the Alexander's model still is one of the best available and applied models. The main idea for theoretical model presented in this paper is on the base of Alexander's model. However other assumptions related to problem have been added. At the end, mean load and absorbed energy are presented. These two relations have corrugation's length and depth as two independent variables. Schematic diagram of cross section of corrugated tube is presented in below figure.
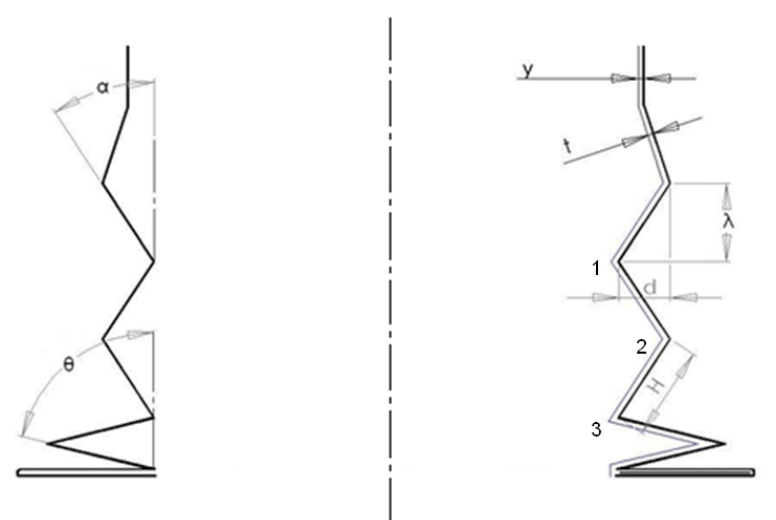

Figure 1. Schematic diagram of cross section of corrugated tube. 
The needed energy to force for plastic deformation consists of two parts. First is "bending energy" in four plastic hinges. It should be considered that the hinge number 2 (with variable radius) rotate two times than the hinge number 1 and number 3 . Hence it is considered two hinges which are rotating 90 degree. Second is "membrane energy" for two arms consisting 1-2 and 2-3 (Fig.1).

A. Bending Energy: bending energy is represented with $\mathrm{E}_{\mathrm{b}}$ and membrane energy is represented with $\mathrm{E}_{\mathrm{s}}$. Bending energy is calculated as follow formula:

$$
E_{b 1,3}=\int_{\alpha}^{\frac{\pi}{2}} 2 M_{0} \pi\left(2 R_{0}\right) d \theta
$$

Where:

- $\quad \alpha$ is bending angle

- $\mathrm{M}_{0}$ is the plastic bending moment, which is:

$$
M_{0}=\left(2 \frac{\sigma_{0}}{\sqrt{3}}\right) \frac{t^{2}}{4}
$$

In the similar way, the bending energy for hinge number 2 is calculated as below:

$$
E_{b 2}=\int_{\alpha}^{\frac{\pi}{2}} 2 M_{0} \pi 2\left(R_{0}+H \sin \theta\right) d \theta
$$

Hence by calculating two above formulas, it is obtained:

$$
\begin{aligned}
E_{b} & =\int_{\alpha}^{\frac{\pi}{2}} 8 M_{0} \pi R_{0} d \theta+\int_{\alpha}^{\frac{\pi}{2}} 4 M_{0} H \sin \theta d \theta \\
& =8 M_{0} \pi R_{0}\left(\frac{\pi}{2}-\alpha\right)+4 M_{0} \pi H \cos \theta
\end{aligned}
$$

B. Membrane Energy: membrane energy (energy required for stretching metal between hinges) in arms 1-2 and 2-3 (fig. 1):

$$
E_{s}=2 \int \sigma_{0} \varepsilon_{s} d v
$$

Where:

- Real amount of strain is:

$$
\begin{aligned}
& \varepsilon_{s}=\ln \left(\frac{R+x \sin \theta}{R}\right) \text { which can be } \\
& \text { assumed } \frac{x \sin \theta}{R} .
\end{aligned}
$$

Then, the membrane energy will be:

$$
\begin{aligned}
E_{s} & =2 \int_{0}^{H} \sigma_{0} \frac{x \sin \theta}{R} t 2 \pi(R+x \sin \theta) d x \\
& =2 \sigma_{0} \frac{\sin \theta}{R} t 2 \pi \int_{0}^{H} x(R+x \sin \theta) \\
& =2 \sigma_{0} \frac{\sin \theta}{R} t 2 \pi\left[\frac{R H^{2}}{2}+\frac{H^{3}}{3} \sin \theta\right]
\end{aligned}
$$

Energy required for deforming corrugated tube can be obtained by taking the required energy for producing corrugated tube from required energy to complete deformation as follow:

$$
E_{s \alpha}=\frac{4 \pi \sigma \sin \theta t}{R}\left(R \frac{H^{2}}{L}+\frac{H^{3}}{3} \sin \alpha\right)
$$

In which, $\alpha$ is corrugation angle.

$$
E_{s \frac{\pi}{2}}=\frac{4 \pi \sigma t}{R}\left(R \frac{H^{2}}{2}+\frac{H^{3}}{3}\right)
$$

Difference of two above relations result is:

$$
\begin{aligned}
E_{s} & =E_{s \frac{\pi}{2}}-E_{s \alpha} \\
& =\frac{4 \pi \sigma t}{R}\left(R \frac{H^{2}}{L}(1-\sin \alpha)+\frac{H^{3}}{3}\left(1-\sin ^{2} \alpha\right)\right)
\end{aligned}
$$

In all above relations, it is assumed that the convolutions are formed externally. But for an alternative mode of collapse, convolutions are formed internally. We have the final result for this case is:

$$
\begin{aligned}
& E_{b}=8 M_{0} \pi R_{0}(\pi / 2-\alpha)-4 M_{0} \pi H[\cos \alpha] \\
& E_{s}=\frac{4 \pi \sigma_{0} t}{R}\left(R \frac{H^{2}}{2}(1-\sin \alpha)-\frac{H^{3}}{3}\left(1-\sin ^{2} \alpha\right)\right)
\end{aligned}
$$

By adding two relations for internal and external convolution, the mean value is:

$$
E=E_{b}+E_{s}=8 M_{0}=R_{0}(\pi / 2-\alpha)+2 \pi \sigma t H^{2}(1-\sin \alpha)
$$

It is worth to mention that this theory assumed half of the convolutions are formed internally and half of them are formed externally which is true and experimental works also prove this.

It is also worth to consider that:

- When $\alpha$ approach to zero, the formula (1) is equal to Alexander's formula 
- When $\alpha$ approach to 900 , it is equal to zero which in both cases are correct.

Equation (1) presents the required energy to complete occurred convolution. In order to obtain equation for mean load we need to calculate the convolution length $(H)$ according to corrugation length $(2 \lambda)$ and corrugation depth $(\mathrm{d})$.

By assuming the cross section between two corrugations is straight line (Fig. 1), the length of convolution will be:

$$
H=\sqrt{\lambda^{2}+d^{2}}
$$

Derived equation for $\alpha$ (initial corrugation angel) with corrugation length $(\lambda)$ and corrugation depth (d) as independent variable is:

$$
\alpha=\operatorname{Arctan}\left(\frac{d}{\lambda}\right)
$$

Besides, in fabrication process, wall of tube had been stretched, it leads to the reduction of wall thickness. Assume that the volume is constant and initial wall thickness (before fabrication of corrugation) is (y), following equation is applicable:

$$
\lambda y=H t \Rightarrow t=\frac{\lambda y}{\sqrt{\lambda^{2}+d^{2}}}
$$

From equation (1) and (2), the total energy becomes:

$E=8\left(\frac{2}{\sqrt{3}} \frac{\sigma t^{2}}{4}\right) \pi R_{0}\left(\frac{\pi}{2}-\operatorname{Arctan}\left(\frac{d}{\lambda}\right)\right)+2 \pi \sigma t\left(\lambda^{2}+d^{2}\right)\left[1-\sin \left(\arctan \left(\frac{d}{\lambda}\right)\right)\right]$

In equation, by assuming $\sigma 0$ is constant, absorbed energy can be calculated in relation with corrugation length $(\lambda)$ and corrugation depth $(d)$.

For calculating mean load, it is possible to make the work done by external forces equal to work done by internal plastic deformation. The final result becomes:

$$
P=\frac{8\left(\frac{2}{\sqrt{3}} \frac{\sigma t^{2}}{4}\right) \pi R_{0}\left(\frac{\pi}{2}-\operatorname{Arctan}\left(\frac{d}{\lambda}\right)\right)+2 \pi \sigma t\left(\lambda^{2}+d^{2}\right)\left[1-\sin \left(\arctan \left(\frac{d}{\lambda}\right)\right)\right]}{2 \lambda}
$$

Theoretical equation obtained here shows the changes of mean load along with changing of corrugation dimension. In other word, by knowing precise value of yielding stress, we can predict the mean load in terms of corrugation parameters.

\section{Conclusion}

Predicting mean load or designing of energy absorbers are essential. Engineers and scientists are always suggesting new theoretical model to calculate this mean load. Currently, many models are available to predict simple tubes without corrugation. Alexander's model is an example of these theoretical models. In every new theoretical model, scientists try to reduce the simplification assumption and increase the accuracy of prediction. Alexander's theoretical model which is one of the initial models has high accuracy and use energy method to calculate mean load.

In this paper, we have presented a theoretical model which is used to predict mean load in corrugated tube on the basis of Alexander's model. Presented model can help designer to have initial prediction of corrugate energy absorbers. This model is obtained by adding assumption to Alexander's model. These assumptions are related to corrugation geometries. Final equation is prediction mean load and absorbed energy.

In final equation independent parameters are dividing in two groups. Firstly, geometrical parameters such as: tube wall thickness, tube diameter, corrugation dimension (length and depth of corrugation)... Secondly, parameters related to material properties like: yielding stress for aluminum... In order to validate theoretical model with experimental ones, thickness and diameter of tube considered as constant values.

This prediction with good accuracy can support designer to design corrugated tubes better.

\section{References}

1. A. G. Mamalis, D. E. Manolakos, G. A. Demosthenous, W. Johnson, Int. J. Impact Eng. 11(2), 185-196 (1991)

2. N. Jones, In: Rossmanith HP, editor. Structural failure, product liability and technical insurance. Amsterdam: Elsevier, 39-48 (1993)

3. N. Jones, Mech. Rev. 42(4), 95-115 (1989)

4. Z. M. Xie, Z. M. Wan, H. W. Song, X. W. Du, Proceeding of ACCM-1, Osaka, Japan, October p. 738.1-4 (1998)

5. A. A. A. Alghamdi, Thin- walled Struct. 39, 189 213 (2001)

6. B. Skocezen, J. Skizypek, Int. J. MechSci. 34, 90116 (1992)

7. A. A. Singace, H. El-Sobky, Int. J. MechSci. 39, 249-68 (1997)

8. S. J. Hosseinipour, G. H. Daneshi, Thin-walled Struct. 41, 31-46 (2003)

9. A. M. Elgalai, E. Mahdi, A. M. S. Hamouda, B. S. Sahari, Compos. Struct. 66, 665-71 (2004)

10. E. F. Abdewi, S. Sulaiman, A. M. S. Hamouda, E. Mahdi, Thin-walled Struct. 46, 320-32 (2008)

11. A. Eyvazian, I. Akbarzadeh, M. Shakeri, Procs ImechE Part L: Journal of Materials: Design and Applications 226: 109-118 (2012) 